\title{
Lessons learned in Central Ghana regarding the pandemic influenza a vaccine and how they could be used conserving
} new ebola virus vaccine

\author{
Dennis Adu-Gyasi*, Dennis Konadu Gyasi and Ayamba Samuel \\ Kintampo Health Research Centre, P. O. Box 200, Kintampo North, Ghana
}

\begin{abstract}
Background: The emergence of influenza A (H1N1) virus prompted the development of influenza A (H1N1) monovalent vaccines (2009-H1N1). The use of the vaccine was recommended by the Center for Disease Control and Prevention Advisory Committee on Immunization Practices. Adverse events after vaccinations occur but are generally rare. The study was carried out to identify reported adverse and serious adverse events after administration of an influenza A (H1N1) vaccine.

Method: This cross-sectional study was carried out between mid of July 2010 to end of August 2010 in Kintampo North Municipality and Offinso South Municipality in Ghana. The study was carried out as part of a pharmacovigilance study in Kintampo Health Research Centre (KHRC). Data were collected from consented participants using questionnaire.

Results: Of the 420 forms that were given out to consented participants in the two regions, 379 (90.2\%) were returned with completed information related to the Influenza A (H1N1) vaccine. Participants who took the vaccine reported of adverse events such as fever, headache, chills, stomach ache, diarrhoea, pain in the heart and fast heartbeat.

Of those who received the vaccine, $4.4 \%$ (16/366) were hospitalized for the adverse event they reported to have experienced after vaccination.

Of the $4.4 \%$ of the vaccinated participants that were hospitalized, $43.8 \%(7 / 16)$ were males and $56.3 \%(9 / 16)$ were females. There was no difference between the proportions $(\mathrm{p}=0.97)$ of males and females that were hospitalized after vaccination.

Conclusion: Ghana used Pandemrix- Influenza A (H1N1) vaccine in June 2010. Symptoms reported ranged from what were expected to unexpected symptoms. This survey recorded sixteen (16) hospitalizations due to symptoms reported after vaccination. The study was not controlled and therefore could not make claims of whether the serious adverse events were associated with the vaccine. We recommend continuous and effective post-vaccination and post-marketing monitoring of adverse events after vaccinations.
\end{abstract}

\section{Introduction}

Emerging diseases of animal origin are a growing concern in the public health. About $75 \%(132 / 175)$ of species of pathogens classified as emerging are zoonotic [1]. Severe acute respiratory syndrome (SARS), avian influenza and Ebola Virus Disease (EVD) which have emerged on a global scale are some of the diseases associated with these pathogens.

The novel H1N1 influenza A virus that emerged in humans in Mexico in April 2009 and transmitted efficiently in the human population according to the World Health Organization (http://www. who.int/csr/don/2009_05_25/en/) spread globally to 46 countries with 12,515 reported cases and 91 associated deaths [2]. On 11 June 2009, the WHO raised the pandemic alert from Level 5 to Level 6 and declared the start of an influenza pandemic [3]. Declaration of the pandemic commenced discussion worldwide among public health officials, governments and pharmaceutical companies about development of an H1N1 vaccine [4].

From the sequenced genomes of samples of the new H1N1 taken from human patients diagnosed with the infection, analysis showed that the H1N1 virus had similar antigenic properties to some existing viral strains that made it easier to come up with a new candidate virus for vaccine development [5]. Vaccine was seen as one of the important means to curb the pandemic [6].
Vaccines serve two related though different functions: to protect vaccinated people against infection and severe disease; and to reduce transmission, thereby offering indirect protection to those not vaccinated via herd immunity [7]. Vaccines most effectively reduce transmission if they are given to the groups that are most likely to be infected and most likely to transmit the infection onward $[7,8]$.The emergence of influenza A (H1N1) virus prompted the development of influenza A (H1N1) monovalent vaccines (2009-H1N1) which its use after it has been licensed by the Food and Drugs Administration, was recommended by the Centers for Disease Control and Prevention (CDC) Advisory Committee on Immunization Practices (ACIP) $[9,10]$. Influenza A (H1N1) monovalent vaccine (2009-H1N1) was the most effective measure to control the spread of the virus associated with H1N1 Influenza and to reduce its associated morbidity and mortality $[10,11]$. Vaccines which have side effects and differ in effectiveness are usually administered to healthy individuals trusting in its safety

${ }^{\star}$ Correspondence to: Dennis Adu-Gyasi, Kintampo Health Research Centre, P. O. Box 200, Kintampo North, Ghana, E-mail address: adugyasidennis@yahoo.co.uk

Key words: Serious adverse event, adverse event, hospitalization and symptoms

Received: April 20, 2018; Accepted: May 10, 2018; Published: May 14, 2018 
and efficacy. Most vaccines have contributed substantially to improve human health in the past century.

The timing of vaccine production and release is a crucial factor. Development of the pandemic vaccine for emergency use also required some flexibility in the regulatory pathway because time was not sufficient for full clinical trials [7-12]. The challenge is that between development of a vaccine and its use publicly, there is a multiplicity and heterogeneity of human factors that must be negotiated to realise the public health gains of using the vaccine to immunize [13].

Ideally, vaccines manufactured after pre-clinical trials are to be taken through three phases of clinical trials to assess safety, efficiency, immunogenicity and efficacy prior to marketing approval. This allows the evaluation to be carried out among individuals of varying traits and larger population. The Influenza A H1N1 pandemic vaccine received approval for use after assessment of the convincing results of ongoing clinical trials $[14,15]$. This can be understood considering the need for a vaccine in the pandemic because the development of the Influenza A H1N1 pandemic vaccine was re-engineered based on an existing vaccine for H5N1 virus strain using the 'Mock-up' approach [10-16]. Hence might not have required the full length of phases in clinical trials. When making decisions about regulatory approval and public health use of vaccines, authorities need to be convinced that the benefits to reduce disease outweigh actual and potential risks of vaccination [17].

Adverse events after vaccinations occur but are generally rare. Some adverse events are unlikely to be detected in pre-licensure clinical trials because of their low frequency, the limited numbers of enrolled subjects, and other study limitations $[18,19]$. These limitations may hinder the reports of serious adverse events during the clinical trials of vaccines.

Readers' comments and responses on specific stories provided by media outlets and to respond to other readers' comments serve as a gauge of public opinion that is immediate, spontaneous and (presumably) honest [4].

Pandemic Influenza A H1N1 2009 was first recorded in Ghana in October, 2009 [20,21]. Media reportage of the pandemic heightened as there were threats of cases being found and diagnosed in Ghana. In the 2010 half year report of the Ashanti Regional Health Directorate of the Ghana Health Service, an H1N1 outbreak was reported in a in primary school in the capital city [22]. Due to the number of vaccines received, when the Ministry of Health decided to vaccinate Ghanaians against the influenza, it was limited and was targeted at health workers, persons at risk of severe disease, security personnel and schools that were reported to have suspected cases $[20,21]$. The H1N1 pandemic Influenza vaccination which recorded excellent coverage received a lot of negative reports from the media and rumours of severe adverse reactions $[22,23]$.

Ebola Virus Disease (EVD) Outbreak in West Africa (Guinea, Liberia, Sierra Leone, Nigeria, Mali and Senegal) was notified as extraordinary event \& Public Health Emergency of International Importance (PHEIC) in August, 2014 by WHO [24].

The situation raised fundamental questions on: how much emphasis should the international community place on experimental interventions in response to the Ebola epidemic; what were the ethical considerations if experimental treatments or vaccines were deployed; if any interventions proved safe and effective, how can they be made more widely available (Rid and Emanuel, 2014), and the cause of the delay with which the international community reacted to the EVD menace [25].
The plague of the EVD was in an unprecedented manner and killed most of its infected victims since April 2014 [26]. The quest to curb the menace made health regulatory authorities give directives in medical practice that were questioned ethically. This was obvious in the permit given for the Z-MAPP to be used for the treatment of Ebola infected individuals $[27,28]$. Vaccines have been developed and are already in the processes for clinical trials for possible registration and usage. We report of identified adverse and serious adverse events that were recorded in a survey to assess an influenza $\mathrm{A}(\mathrm{H} 1 \mathrm{~N} 1)$ vaccine introduced and administered in the public health system of Ghana to handle the emergence of the pandemic in Ghana from June 2010 to September 2010. This in our view will present lessons that need to be considered in the processes of taking possible Ebola vaccines through clinical trials, approvals and marketing.

\section{Method}

Descriptions: Vaccine: Pandemrix vaccine is a monovalent, split virion inactivated influenza vaccine containing antigen from $\mathrm{A} /$ California/07/2009 (H1N1) v strain [29]. It contains an immunologic adjuvant AS03 which consists of DL- $\alpha$-tocopherol (vitamin E), squalene and polysorbate 80 , with Thiomersal (thimerosal) as preservative with additional important non-medicinal ingredients such as formaldehyde, sodium deoxycholate, and sucrose. The final formulation contains $3.75 \mu \mathrm{g}$ haemagglutinin (HA) per $0.5 \mathrm{ml}$ dose adjuvanted with AS03. The vaccine is given as a single dose for most people, with a two-dose schedule for children under the age of 10 years and immunocompromised adults $[29,30]$.

Adverse event: Any symptom a participant experienced after the administration of the pandemic influenza vaccine.

Serious Adverse event: In the period of the survey, we considered any symptom a participant experienced after the administration of the pandemic influenza vaccine, reported and was hospitalized to stabilise.

Study design and site: The cross-sectional study was carried out between mid of July 2010 and 31st of August 2010 in the Kintampo North and Offinso South Municipality in the Brong Ahafo and Ashanti Regions of Ghana respectively.

Kintampo North Municipal cover in total, an area of 5,108km2 with a resident population of approximately 96,358 (Ghana Districts). The study area is located within the forest-savannah transitional ecological zone in Ghana where subsistent farming is the predominant occupation. Mean monthly temperatures range between $18^{\circ} \mathrm{C}$ to $38^{\circ} \mathrm{C}$ and rainfall averages $1250 \mathrm{~mm}$ per annum. Since 2010 the study area has records of average vaccination coverage of at least $90 \%$ of the various vaccines in the Expanded Programme on Immunization (EPI).

Offinso South Municipal is located in the extreme north-western part of the Ashanti Region. Offinso Municipal lies between longitude 1' $65 \mathrm{~W}$ and 1' $45 \mathrm{E}$ and latitudes 6' $45 \mathrm{~N}$ and 7' $25 \mathrm{~S}$. The municipal covers an area of $1255 \mathrm{~km} 2$ with a resident population of approximately 138,190 . The municipality had an average EPI coverage of at least $80 \%$ in 2010 and also an H1N1 vaccination coverage of about $62 \%$ of the target population during the period of vaccination [31].

Study Population: The study team distributed questionnaire to consented participants who could read and understand English with minimum instructions and introduction since the questionnaire were written in English. Aside this criterion, individuals targeted to be interviewed were to be more than fifteen (15) years of age. Questionnaires were therefore distributed to prospective participants 
assumed to be at the level of Senior High School and beyond (Midwifery/ Nursing Training and Teacher Training Institutions) based on age for interviewing. The selection of the population was made to reflect the group of individuals that were considered by the Ministry of Health to be at high risk and were considered for the vaccination.

\section{Procedures}

Members of the study team piloted the administration of the questionnaire which was adopted from the tools use for the Safety and Pharmacovigilance component of a study in the Kintampo Health Research Centre from the Food and Drugs Authority, Ghana with information from the CDC website on known symptoms associated with influenza A H1N1 infection. Information from the media reports were also used to modify the questionnaire to find out if any unsuspected adverse event could be found among individuals vaccinated with Pandemrix.

Mode of administration of the questionnaire was regularised in order to obtain data that would be comparable since we were collecting and collating data from two different regions of the country.

The study was introduced and explained to individuals, groups of people who were considered eligible. Inform consents were given by individuals who signed to respond to the questionnaires administered.

Ethical Issues: Informed consent was sought from all participants in the study after a detailed explanation of the purpose of the study. Permissions were also sought from the heads of the various institutions that were used for the study. The survey was approved by the Institutional Ethics Review Committee of Kintampo Health Research Centre, Ghana.

Data Management and Analysis: Microsoft Access data management software version 2007 was used for data entry. Stata software version 12 and GraphPad Prism software version 5.0 were used for statistical analyses of the data. All data were double entered on computers, range and consistency checks were performed and discrepancies resolved with reference to the original form. The proportions of participants who reported adverse events after vaccination were tabulated. Differences in symptoms reported among hospitalized and those not hospitalized after vaccination were considered significant with $95 \%$ confidence interval.

\section{Results}

Of a total of 420 forms that were given out to consented participants in the two regions, $379(90.2 \%)$ were returned with completed information related to the Influenza A (H1N1) vaccine. The number of participants from each of the two regions where the survey was carried out were equally distributed with 197 (52.0\%) from the Brong Ahafo and $182(48.0 \%)$ from the Ashanti Regions of Ghana.

Of a total of 367 data obtained on the sex participants, 166 (45.2\%) were males with 201 (54.8\%) being females. The entire population were in the age range of 17 years to 66 years with a median age of 21years. The levels of education of the participants extended from primary through secondary to tertiary. Students (75.7\%) formed the bulk of the population interviewed with $19.7 \%$ being prospective health workers (Table 1).

Vaccination coverage among the participants was as high as $366 / 379$ (96.6\%). Participants who received the vaccine reported of adverse events ranging from known (what has been described to be associated with the vaccine as presented by the $\mathrm{CDC}$ ) and reported such as fever,
Table 1. Description of the demographic characteristics of the population in the study

\begin{tabular}{|l|l|l|l|}
\hline Age (Years) & Observations & Mean(S.D) & n (\%) Missing \\
\hline Sex & 342 & $22.9(6.1)$ & $37(9.8)$ \\
\hline EDUCATION & $\begin{array}{l}\mathrm{n}(\%) \text { Males }=166 \\
(43.8)\end{array}$ & $\begin{array}{l}\mathrm{n}(\%) \text { Females) } \\
201(53.0)\end{array}$ & $\begin{array}{l}\mathrm{n}(\%) \text { Missing } \\
12(3.2)\end{array}$ \\
\hline Primary & $\mathrm{n}(\%)$ & \\
\hline Secondary & $5(1.3)$ & & \\
\hline Tertiary & $192(50.7)$ & & \\
\hline OCCUPATION & $182(48.0)$ & & \\
\hline Students (at least High & $\mathrm{n}(\%)$ & \\
\hline School) & $287(75.7)$ & & \\
\hline Health workers & $75(19.8)$ & & \\
\hline Teachers & $12(3.2)$ & & \\
\hline Other Profession & $5(1.3)$ & & \\
\hline Total & $379(100)$ & & \\
\hline
\end{tabular}

headache, chills, stomach ache and diarrhoea to unknown (what was not found to be listed to be associated to the pandemix influenza vaccine) and unreported such as pain in the heart, fast heartbeat and others presented in table 2 (Table 2).

Hospitalization, a serious adverse event, of $4.4 \%$ (16/366) was recorded among the participants who received the vaccine for the adverse events reported after vaccination. Majority, 56.3\% (9/16), of the hospitalized individuals stayed on admission for three (3) days with only one person who had a prolonged stay of seven (7) days in hospital (Figure 1).

Of the $4.4 \%$ of the vaccinated participants that were hospitalized, $43.8 \%(7 / 16)$ were males and $56.3 \%$ (9/16) were females. There was no significant difference $(p=0.97)$ between the proportions of males and females that were hospitalized after vaccination.

In total, $68.3 \%$ (250/379) of the participants responded to the question on the duration it took for an adverse event experienced after vaccination to resolve. The duration a participant endured an adverse event ranged from a minimum of one day to a maximum of twenty days (Table 3 ).

\section{Discussions}

Ghana started use of Pandemrix- Influenza A (H1N1) vaccine in June 2010. Upon its introduction, there was a lot of public outcry about the adverse events experienced after vaccination. The heightened media attention increased the awareness of the public to the potential vaccine side effects. The symptoms observed from the participants in multiple responses after vaccination that were heightened ranged from "severe pain at injection site" (12.3\%), "headache" (11.7\%), "fever" (10.3\%) and "General body pains" (9.0\%) to unexpected "fast-heart beat" $(2.0 \%)$ and "pain in heart" $(0.7 \%)$ adverse events (Table 2). Notably, with the emerging suspicion that Pandemrix could be linked to narcolepsy, the survey recorded $4.7 \%$ of drowsiness (feeling sleepy) and $4.7 \%$ of sleeplessness [32,33].

This survey of 379 people considered to be in a high-risk group to receive the pandemrix recorded sixteen (16) hospitalizations due to symptoms reported after vaccination. The study was not controlled and therefore could not make claims of whether the serious adverse events were associated with the vaccine. However, it is interesting to note the prolong hospitalization was recorded of individuals that received the vaccine and were hospitalized for the adverse events they developed.

The study was not designed to do proper systematic causal assessments of the serious adverse events and therefore does not have 
Table 2. Frequently reported adverse event associated with influenza A (H1N1) 2009 vaccine in Central Ghana

\begin{tabular}{|c|c|c|}
\hline Adverse event & $\mathrm{N}$ (\% of responses) & $\%$ of cases \\
\hline Fever & $148(10.3)$ & 40.7 \\
\hline Headache & $168(11.7)$ & 46.2 \\
\hline Fatigue & $116(8.1)$ & 31.9 \\
\hline Vomiting & $13(0.9)$ & 3.6 \\
\hline Nausea & $34(2.4)$ & 9.3 \\
\hline Stomach ache & $23(1.6)$ & 6.3 \\
\hline General body pains & $139(9.7)$ & 38.2 \\
\hline Swelling at injection site & $28(2.0)$ & 7.7 \\
\hline Diarrhoea & $20(1.4)$ & 5.5 \\
\hline Inflammation at site & $12(0.8)$ & 3.3 \\
\hline Itching & $22(1.5)$ & 6.0 \\
\hline Dizziness & $48(3.4)$ & 13.2 \\
\hline Drowsiness & $68(4.7)$ & 18.7 \\
\hline Sleeplessness & $68(4.7)$ & 18.7 \\
\hline Loss of appetite & $56(3.9)$ & 15.4 \\
\hline Chills & $77(5.4)$ & 21.2 \\
\hline Restlessness & $48(3.4)$ & 13.2 \\
\hline Stuffy nose & $21(1.5)$ & 5.8 \\
\hline Fast heart-beat & $28(2.0)$ & 7.7 \\
\hline Pain in heart & $10(0.7)$ & 2.8 \\
\hline Severe pain at injection site & $176(12.3)$ & 48.4 \\
\hline Runny nose & $34(2.4)$ & 9.3 \\
\hline Others & $8(0.6)$ & 2.2 \\
\hline None & $69(4.8)$ & 19.0 \\
\hline Total & $1434(100)$ & 394.0 \\
\hline
\end{tabular}

*Valid cases: 364 participants vaccinated with H1N1 influenza vaccine with multiple responses to symptoms experienced.

Table 3. Indication of the number of days participants experienced the side effect after vaccination

\begin{tabular}{|c|c|}
\hline Duration for AE to clear & N (\% of responses) \\
\hline $\mathbf{1 - 3}$ days & $128(51.2)$ \\
\hline 4-6 days & $75(30.0)$ \\
\hline $\mathbf{7 - 9}$ days & $29(11.6)$ \\
\hline $\mathbf{1 0 - 2 0}$ days & $18(7.2)$ \\
\hline Total & $250(100)$ \\
\hline
\end{tabular}

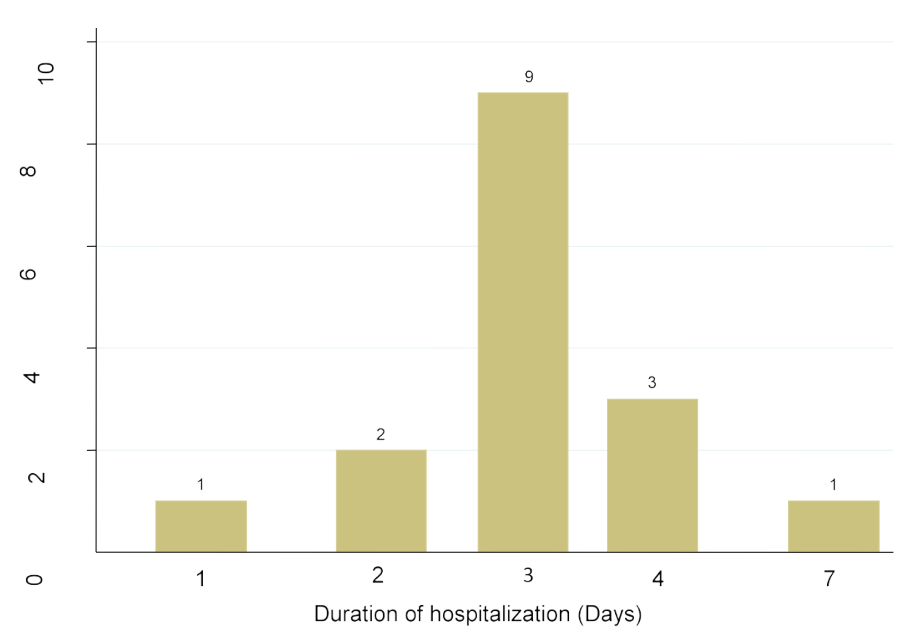

Figure 1. Number of days of hospitalization of vaccinated participants

the power to suggest and claim that the adverse events recorded in the interviews were related to the vaccine administered. Folders of hospitalized participants were not further reviewed for their clinical history and other possible cause of their hospitalizations.
Participants were selected to minimize bias by simply selecting participants who were ready to consent and respond to our questionnaire, providing a control group for better analysis would have minimized speculations and assumptions.

The study had a limitation of selection bias. Since the study was interested to source information on adverse and serious adverse events after vaccine administration, participants' selection was from high risk individuals who had been considered for vaccination with the $\mathrm{H} 1 \mathrm{~N} 1$ Influenza Pandemrix vaccine.

Also, there was the possibility of the effect of recall bias and the ability of participants to understand the various checklist of symptoms presented on the questionnaire. Though the symptoms were presented in very simplified English language, the study did not assess the level of participants understanding of the symptoms which could also affect what was chosen as response.

\section{Lessons to EBV vaccines}

Effectiveness data if available would be necessary to perform a clear and fair risk-benefit balance assessment; this assessment is important taking into account the characteristics of the $\mathrm{H} 1 \mathrm{~N} 1$ influenza pandemic [9-34]. Pharmaceutical companies have produced vaccines to EVD and the desire to take it through clinical trials and further use for protection if found efficacious. These gave rise to ethical, safety and scientific concerns in the manner in which the processes to conduct the clinical trials were being hastened [35-36]. It is of good motive these processes were being done to satisfy the quest for vaccine to the plagues, many of which were of zoonotic origin.

We recommend continuous and effective post-vaccination and post-marketing monitoring of adverse events after the introduction of any of the Ebola vaccines to monitor safety and review spontaneously reported adverse events which will add to safety data that will emerge from other sources.

\section{Authors' contributions}

DAG, DKG and KP designed the study. DAG, DKG, KP, LA, $\mathrm{AK}, \mathrm{SA}$ performed the experiments and co-wrote the manuscript. $\mathrm{KB}$ supported with the data collection. EM, AM and SOA contributed to the study design, carried out the statistical analysis, performance of experiments and wrote the manuscript. DAG, KP, SOA designed the experiments, supervised the study and co-wrote the manuscript.

\section{Acknowledgements}

The Director and staff of KHRC for their guide and support to develop this work. The heads of the institutions that allowed the questionnaire to be administered are greatly appreciated. Particular mention will be made of Kintampo Senior High School. Offinso Teacher's Training College, Kintampo Municipal Hospita and St. Patrick's Hospital, Offinso. Our gratitude is expressed for the role Mr. Kwadwo Boateng during data collection and Miss. Ethel Amoah during data entry.

\section{Conflict of Interest}

The authors declare no conflict of interest.

\section{Funding}

Funding support was given by Kintampo Health Research Centre. 


\section{References}

1. Leslie MJ, Mcquiston JH (2007) Surveillance for zoonotic diseases. Infectious Disease Surveillance 93-106.

2. Poon LL, Chan K, Smith G, Leung C, Guan Y, et al. (2009) Molecular detection of a novel human influenza (H1N1) of pandemic potential by conventional and real-time quantitative RT-PCR assays. Clinical Chemistry 55: 1555-1558.

3. Brockwell-Staats C, Webster RG, Webby RJ (2009) Diversity of influenza viruses in swine and the emergence of a novel human pandemic influenza A (H1N1). Influenza and other respiratory viruses 3: 207-213.

4. Henrich N, Holmes B (2011) What the public was saying about the H1N1 vaccine: perceptions and issues discussed in on-line comments during the $2009 \mathrm{H} 1 \mathrm{~N} 1$ pandemic. PLoS One 6: e18479. [Crossref]

5. Greninger AL, Chen EC, Sittler T, Scheinerman A, Roubinian N, et al. (2010) A metagenomic analysis of pandemic influenza A $(2009 \mathrm{H} 1 \mathrm{~N} 1)$ infection in patients from North America. PLoS One 5: e13381. [Crossref]

6. Ferguson NM, Cummings DA, Fraser C, Cajka JC, Cooley PC, et al. (2006) Strategies for mitigating an influenza pandemic. Nature 442: 448-452. [Crossref]

7. Smith J, Lipsitch M, Almond JW (2011) Vaccine production, distribution, access, and uptake. Lancet 378: 428-438. [Crossref]

8. Backer JA, Hagenaars TJ, Van Roermund HJ, De Jong MCM (2009) Modelling the effectiveness and risks of vaccination strategies to control classical swine fever epidemics. Journal of The Royal Society Interface 6: 849-861.

9. Girard MP, Tam JS, Assossou OM, Kieny MP (2010) The 2009 A (H1N1) influenza virus pandemic: A review. Vaccine 28: 4895-4902. [Crossref]

10. Greenberg ME, Lai MH, Hartel GF, Wichems CH, Gittleson C, et al. (2009) Response to a monovalent 2009 influenza A (H1N1) vaccine. $N$ Engl J Med 361: 2405-2413. [Crossref]

11. Luke CJ, Lakdawala SS, Subbarao K (2012) Influenza vaccine-live. Vaccines: Expert Consult-Online 294.

12. Nagurney A (2011) Risk Perceptions, Past Vaccination, and Vaccine Acceptance for Seasonal and Outbreak (2009 H1N1) Influenzas Among a University Sample. Texas State University.

13. Moxon ER, Das P, Greenwood B, Heymann DL, Horton R, et al. (2011) A call to action for the new decade of vaccines. Lancet 378: 298-302. [Crossref]

14. G.S.K (2009) Pandemic 2009 Influenza Update: US FDA approves GSK's pandemic H1N1 vaccine. Press Release 11 November 2009. Retrieved 12 April 2015.

15. EMA (2009) European Medicines Agency recommends authorisation of two vaccines for influenza pandemic (H1N1) 2009" (Press release). European Medicines Agency. Archived from the original on 7 October 2009. Retrieved 12 April 2015.

16. Garten RJ, Davis CT, Russell CA, Shu B, Lindstrom S, et al. (2009) Antigenic and genetic characteristics of swine-origin 2009 A (H1N1) influenza viruses circulating in humans. Science 325: 197-201.

17. Pfeifer D, Alfonso C, Wood D (2010) Defining the safety profile of pandemic influenza vaccines. Lancet 375: 9-11. [Crossref]
18. Burwen DR, La Voie L, Braun MM, Houck P, Ball R (2007) Evaluating adverse events after vaccination in the Medicare population. Pharmacoepidemiology and drug safety 16: 753-761.

19. Ortqvist A, Blennow M, Carlsson RM, Hanson L, Lindberg A, et al. (2010) Vaccination of children-a systematic review. Acta Paediatrica 99: 1-192.

20. Asiedu-Bekoe F, Adu DA, Offei A (2012) Mass Oseltamivir Prophylaxis Halts Pandemic Influenza a H1N1 2009 Outbreak in a Secondary School in Ashanti Region, Ghana. Ghana Medical Journal 46: 219-224.

21. GHS (2010) C Update on current situation: pandemic in?uenza H1N1 in Ghana.

22. GHS (2010) A Ashanti Region-Regional Health Directorate, Ashanti Regional Report Half Year-2010.

23. Ankrah DN, Mantel-Teeuwisse AK, De Bruin ML, Amoo PK, Ofei-Palm CN, et al (2013) Incidence of Adverse Events Among Healthcare Workers Following H1N1 Mass Immunization in Ghana. Drug safety 36: 259-266.

24. WHO (2014) Disease outbreak news, Ebola.

25. Wolz A (2014) Face to face with Ebola--an emergency care center in Sierra Leone. $N$ Engl J Med 371: 1081-1083. [Crossref]

26. Wilkinson A, Leach M (2014) Briefing: ebola-myths, realities, and structural violence. African Affairs 080.

27. Folayan M, Brown B, Yakubu A, Peterson K, Haire B (2014) Compassionate use of experimental drugs in the Ebola outbreak. Lancet 384: 1843-1844. [Crossref]

28. Donovan GK (2014) Ebola, epidemics, and ethics - what we have learned. Philos Ethics Humanit Med 9: 15. [Crossref]

29. EMC (2013) Pandemrix suspension and emulsion for emulsion for injection summary of product characteristics. Last updated on the eMC 01/Aug/2013. Accessed 13Apr2015.

30. Bias H, Quarcoo D, Meier-Wronski C, Wicker S, Seybold J, et al. (2011) Self-reported adverse reactions in 4337 healthcare workers immunizations against novel H1N1 influenza. BMC research notes 4: 297.

31. GHS (2010) B Kintampo North Municipal Health Directorate, Brong Ahafo Regional Report Half Year Review-2010. Retrieved on 20 November 2010.

32. Nohynek H, Jokinen J, Partinen M, Vaarala O, Kirjavainen T, et al. (2012) AS03 adjuvanted AH1N1 vaccine associated with an abrupt increase in the incidence of childhood narcolepsy in Finland. PloS one 7: e33536.

33. Han F, Lin L, Warby SC, Faraco J, Li J, et al. (2011) Narcolepsy onset is seasonal and increased following the 2009 H1N1 pandemic in China. Ann Neurol 70: 410-417. [Crossref]

34. Jamieson D, Honein M, Rasmussen S, Williams J, Swerdlow D, et al. (2009) H1N1 2009 influenza virus infection during pregnancy in the USA. Lancet 374: 451-458.

35. Rid A, Emanuel EJ (2014) Ethical considerations of experimental interventions in the Ebola outbreak. The Lancet 384: 1896-1899.

36. Smith GJ, Vijaykrishna D, Bahl J, Lycett SJ, Worobey M, et al. (2009) Origins and evolutionary genomics of the 2009 swine-origin H1N1 influenza A epidemic. Nature 459: 1122-1125. [Crossref]

Copyright: (C2018 Adu-Gyasi D. This is an open-access article distributed under the terms of the Creative Commons Attribution License, which permits unrestricted use, distribution, and reproduction in any medium, provided the original author and source are credited. 